\title{
LOCAL COHOMOLOGY MULTIPLICITIES IN TERMS OF ÉTALE COHOMOLOGY
}

\author{
MANUEL BLICKLE AND RAPHAEL BONDU
}

\begin{abstract}
In this paper we give an interpretation of the invariants $\lambda_{a, i}(A)$ introduced by Lyubeznik in Lyu93 for a reasonably general class of singularities. In positive characteristic it is the newly introduced class of close to F-rational varieties and the invariants are described in terms of étale cohomology with $\mathbb{Z} / p \mathbb{Z}$-coefficients. This result presents the first application of Emerton and Kisin's Riemann-Hilbert type correspondence to local algebra. In fact our proof works in characteristic zero as well so that we obtain generalizations of results on these invariants which were previously obtained for isolated singularities by analytic techniques.
\end{abstract}

\section{INTRODUCTION}

Let $(R, m)$ be a regular local ring of dimension $n$ and let $A=R / I$ be a quotient of $R$. In Lyu93 Lyubeznik introduces new invariants $\lambda_{a, i}(A)$ (defined as the ath Bass number of $H_{I}^{n-i}(R)$ ) and shows that if $A$ contains a field, these are independent of the presentation of $A$ as a quotient of a regular local ring. One can verify that

$$
\lambda_{a, i}(A)=e\left(H_{m}^{a}\left(H_{I}^{n-i}(R)\right)\right)
$$

where the multiplicity $e\left(\_\right)$can be described as follows: The main results of Lyu93, HS93] state that the module $H_{m}^{a}\left(H_{I}^{n-i}(R)\right)$ is injective. As it is supported at the maximal ideal it is isomorphic to a finite direct sum of $e$ copies of the injective hull $E_{R / m} \cong H_{m}^{n}(R)$ of the residue field of $R$. This integer $e$ is the multiplicity.

Our main result is the following description of these invariants in the case that $A$ has reasonable singularities.

2000 Mathematics Subject Classification. 14B15,14F20.

Key words and phrases. local cohomology, characteristic $p$, perverse sheaves.

The authors were supported by the DFG Schwerpunkt Globale Methoden in der komplexen Geometrie. 
Theorem 1.1. Let $k$ be a field ${ }^{1}$ of characteristic $p>0$ and let $Y$ be a $k$ variety of dimension d which is close to $F$-rational outside the single (closed) point $x \in Y$. Let $A=\mathcal{O}_{Y, x}$. Then

(1) $\lambda_{0, i}(A)=\operatorname{dim}_{\mathbb{Z} / p \mathbb{Z}} H_{\{x\}}^{i}\left(Y_{\text {ét }}, \mathbb{Z} / p \mathbb{Z}\right)$ for $1 \leq i \leq d-1$.

(2) $\lambda_{a, d}(A)=\operatorname{dim}_{\mathbb{Z} / p \mathbb{Z}} H_{\{x\}}^{d+1-a}\left(Y_{\text {ét }}, \mathbb{Z} / p \mathbb{Z}\right)$ for $2 \leq a \leq d-1$ and $\lambda_{d, d}(A)=$ $\operatorname{dim}_{\mathbb{Z} / p \mathbb{Z}} H_{\{x\}}^{1}\left(Y_{e ́ t}, \mathbb{Z} / p \mathbb{Z}\right)+1$.

(3) All other $\lambda_{a, i}(A)$ vanish.

The notion close to $F$-rational will be introduced and briefly discussed in Section 4. The name is chosen to indicate that $F$-rational varieties are close to $F$-rational and thus so are smooth varieties. In particular, the theorem applies in the case that $Y$ has an isolated singularity at $x$. A key ingredient in our proof is that a close to $F$-rational variety $Y \subseteq X$ ( $X$ smooth) has the property that $\mathbf{R} \Gamma_{[Y]}\left(\mathcal{O}_{X}\right)$ is isomorphic to $\mathcal{L}(Y, X)[d-n]$, the intersection homology module (cf. Section 2).

The isolated singular case was motivated by the main result in GLS98 where García Lpez and Sabbah prove a topological description of the invariants $\lambda_{a, i}(A)$ in the case that $A$ is the local ring of an isolated complex singularity. ${ }^{2}$ Our methods lead to a generalization of their result, replacing the assumption of isolated singularity with the significantly weaker requirement that $(Y-\{x\})$ is an intersection cohomology manifold, i.e. $\mathbb{C}_{(Y-\{x\})}[d] \cong I C_{(Y-\{x\})}^{\bullet}{ }^{3}$

Theorem 1.2. Let $Y$ be a complex variety of dimension $d$ and $x \in Y$ such that $(Y-\{x\})$ is an intersection homology manifold. Let $A=\mathcal{O}_{Y, x}$. Then

(1) $\lambda_{0, i}(A)=\operatorname{dim}_{\mathbb{C}} H_{\{x\}}^{i}(Y, \mathbb{C})$ for $1 \leq i \leq d-1$.

(2) $\lambda_{a, d}(A)=\operatorname{dim}_{\mathbb{C}} H_{\{x\}}^{d+1-a}(Y, \mathbb{C})$ for $2 \leq a \leq d-1$ and $\lambda_{d, d}(A)=$ $\operatorname{dim}_{\mathbb{C}} H_{\{x\}}^{1}(Y, \mathbb{C})+1$.

(3) All other $\lambda_{a, i}(A)$ vanish.

\footnotetext{
${ }^{1}$ As was pointed out by Brian Conrad there is a problem (proof of Lemma 2.7) with our argument (in characteristic $p>0$ ) if $k$ is not separably closed. So " $k$ separably closed" has to be added as an assumption in the theorem - to be safe as an assumption throughout the paper.

However, in general one can reduce to this case. For $k^{\prime}$ the separable closure of $k$ one checks that $\lambda_{a, i}(A)=\lambda_{a, i}\left(A \otimes_{k} k^{\prime}\right)$ as follows: Since tensoring with $k^{\prime}$ over $k$ (which we shall denote by $\left.\left({ }^{\prime}\right)^{\prime}\right)$ is faithfully flat, we have

$$
H_{m^{\prime}}^{a}\left(H_{I^{\prime}}^{n-i}\left(R^{\prime}\right) \cong k^{\prime} \otimes_{k} H_{m}^{a} H_{I}^{n-i}(R)\right.
$$

and $E_{R / m} \cong E_{R^{\prime} / m^{\prime}}$ (using that $E_{R / m} \cong H_{m}^{n}(R)$ ) which implies the claimed equality. Hence, in general, one is still able to reduce to the case proven by our main theorem.

${ }^{2}$ To be precise, they state part (2) in its Poincaré dual form $\left(\lambda_{a, d}=\operatorname{dim}_{\mathbb{C}} H^{d+a}(Y, \mathbb{C})\right.$ for $2 \leq a \leq d)$, see in Remark 2.2 why this is the case and also why we prefer our version.

${ }^{3}$ This notion was recently introduced by Massey Mas and via the Riemann-Hilbert correspondence it is clearly the condition corresponding to $\mathcal{L}(Y, X) \cong \mathbf{R} \Gamma_{[Y]}\left(\mathcal{O}_{X}\right)[n-d]$
} 
In the isolated singular case, statement (1) was already pointed out in Lyu93 to follow from a result of Ogus Ogu73, Theorem 2.3]. Observing the proof in GLS98 we first note that part (3) is independent of the characteristic whereas the other parts distinctively use characteristic zero.

In order to obtain the full analog of Theorem 1.2 in positive characteristic we have to work somewhat harder. The proof given in GLS98 is our point of departure. They use the Riemann-Hilbert correspondence and duality for holonomic $D$-modules. Our idea is to replace the Riemann-Hilbert correspondence (i.e. de Rham theory) with the correspondence recently introduced by Emerton and Kisin EK04 (i.e. Artin-Schreier theory). The main obstacle is that the categories involved in the Emerton-Kisin correspondence do not have a duality, which was an essential part in the proof of García Lpez and Sabbah. Thus our first task is to give a new proof of Theorem 1.2 which as its main feature avoids the use of duality. In this proof we also show explicitly that part (1) and (2) are equivalent once part (3) is established.

In Section 3 we briefly recall the setup for the Emerton-Kisin correspondence and show that this allows us to carry our new characteristic zero proof over to positive characteristic. Thus we obtain Theorem 1.1 postponing the introduction and investigation of close to F-rational singularities to the last section.

\section{Duality free Proof in CHARACTERISTIC ZeRo.}

2.1. A spectral sequence computation. We start with explaining that a vanishing condition (slightly weaker than the one in part (3) of Theorem 1.2) for the $\lambda_{a, i}(A)$ implies part (3) and also that part (1) and (2) are equivalent. This is done via a not so difficult spectral sequence argument. The condition we impose is that

$$
\lambda_{a, i}=e\left(H_{[x]}^{a} H_{[Y]}^{n-i}\left(\mathcal{O}_{X}\right)\right)=0
$$

for all pairs $(a, i)$ with $a>1$ and $i \neq d$. This is, for example, satisfied if $Y$ a complete intersection at $x$. We will then show that (2.1) implies that

$$
\begin{gathered}
\lambda_{0,1}(A)+1=\lambda_{d, d}(A) \quad \text { and } \\
\lambda_{0, d-r+1}(A)=\lambda_{r, d}(A) \text { for } 2 \leq r \leq d-1
\end{gathered}
$$

and that all other $\lambda_{a, i}$ are zero. This clearly suffices to support all our claims. Now consider the spectral sequence

$$
E_{2}^{a, j}=H_{[x]}^{a} H_{[Y]}^{j}\left(\mathcal{O}_{X}\right) \Rightarrow H_{[x]}^{a+j}\left(\mathcal{O}_{X}\right) .
$$

Since $\lambda_{a, i}=e\left(E_{2}^{a, n-i}\right)$ the vanishing assumption (2.1) yields that the only possibly nonzero entries of the $E_{2}$ sheet of this spectral sequence are the 
ones illustrated in the picture:

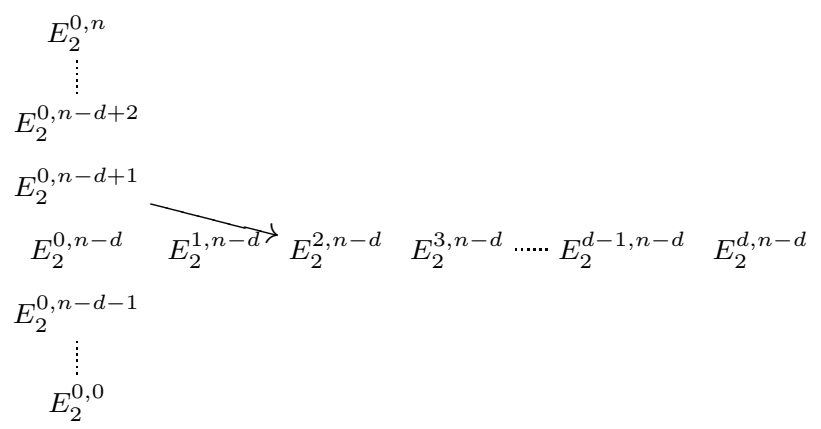

Clearly, the only possibly nonzero arrow is the one indicated. We now assume that $d \geq 2$ and leave the easy cases $d=1$ and $d=0$ to the reader. Recall that by the Hartshorne-Lichtenbaum vanishing theorem one has $H_{[Y]}^{n}\left(\mathcal{O}_{X}\right)=0$ and therefore $E_{2}^{0, n}=H_{[x]}^{0} H_{[Y]}^{n}\left(\mathcal{O}_{X}\right)=0$ which just says that $\lambda_{0,0}(A)=0$. Now we claim that for $r \geq 2$ the $E_{r}$ sheet of the spectral sequence has only the following (possibly) nonzero entries

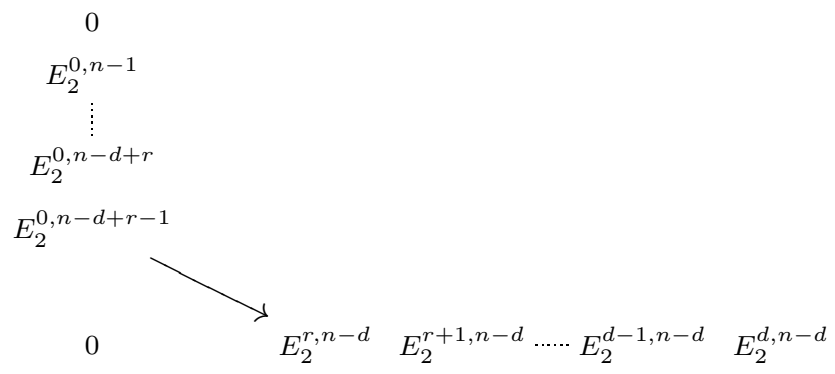

where the only nonzero arrow is the one indicated which yields an isomorphism

$$
H_{[x]}^{0} H_{[Y]}^{n-d+r-1}\left(\mathcal{O}_{X}\right) \cong H_{[x]}^{r} H_{[Y]}^{n-d}\left(\mathcal{O}_{X}\right)
$$

for $r<d$. In the case $r=d$ this only nonzero arrow fits in a short exact sequence

$$
0 \rightarrow H_{[x]}^{0} H_{[Y]}^{n-1}\left(\mathcal{O}_{X}\right) \rightarrow H_{[x]}^{d} H_{[Y]}^{n-d}\left(\mathcal{O}_{X}\right) \rightarrow H_{[x]}^{n}\left(\mathcal{O}_{X}\right) \rightarrow 0
$$

the right map being the edge map of the spectral sequence. ${ }^{4}$ All these claims simply follow from the observation that in the limit of the spectral sequence the only surviving term is $H_{[x]}^{n}\left(\mathcal{O}_{X}\right)$ and the fact that each sheet has only one nonzero arrow. For $r<d$ the bottom left terms (the ones below the nonzero arrow) must vanish since they do not contribute to the limit (the only term that does is $E^{d, n-d}$ ) and since there are no nonzero arrows arriving at or departing from any of them in the $r$ th or any higher sheet. Similarly

\footnotetext{
${ }^{4}$ The injectivity on the left is clear since $E_{2}^{0, n-1}$ must die in the limit. The surjectivity on the right follows since $E_{2}^{0,0}=0$, thus the term in the middle $\left(E_{2}^{d, d}\right)$ is the only one that can contribute to the abutment term, thus has to surject onto it.
} 
the only nonzero arrow must be an isomorphism since otherwise there would be a surviving term in the next sheet which is impossible as we just argued. Rephrasing these observations in terms of the $\lambda_{a, i}(A)$ we obtain from (2.2) that

$$
\lambda_{0, d-r+1}(A)=\lambda_{r, d}(A)
$$

for $2 \leq r \leq d-1$ and from (2.3) that

$$
\lambda_{0,1}(A)+1=\lambda_{d, d}(A)
$$

where we used in the latter that $e\left(\_\right)$is additive on short exact sequences and that $e\left(H_{[x]}^{n}\left(\mathcal{O}_{X}\right)\right)=1$. That all other $\lambda_{a, i}$ vanish follows already form the shape of the $E_{2}$-sheet.

Remark 2.1. The vanishing condition (2.1) is satisfied precisely when $H_{[Y]}^{j}\left(\mathcal{O}_{X}\right)$ is supported at the point $x$ for $j \neq n-d$. This, in turn, clearly holds whenever $Y$ has an isolated singularity at $x$ and is smooth otherwise.

Remark 2.2. In GLS98 García Lpez and Sabbah prove the Poincaré dual statement of part $(2)$, namely that $\lambda_{a, d}=\operatorname{dim}_{\mathbb{C}} H^{d+a}(Y, \mathbb{C})$ for $2 \leq a \leq d .^{5}$ The reason for this lies in their computation of $\lambda_{a, d}(A)$ which uses duality for holonomic $\mathcal{D}$-modules which under Riemann-Hilbert corresponds to Poincaré duality, in that special case. Thus they obtain the equivalence of part (1) and part (2) as a consequence of Poincaré duality. Our observation though shows that this equivalence follows from the structure of the invariants $\lambda_{a, i}$ and the use of Poincaré duality can be avoided.

2.2. Preparatory lemmata and proof of Theorem 1.2. We start with some (probably well known) facts which will naturally lead to the proof of Theorem 1.2

Lemma 2.3. Let $X$ be a smooth $\mathbb{C}$-variety of dimension $n$ and let $k: x \hookrightarrow X$ be the inclusion of a point. Let $\mathcal{M}$ be a holonomic $\mathcal{D}_{X}$-module, then

$$
\operatorname{Sol}\left(H_{[x]}^{a}(\mathcal{M})\right) \cong k_{!} k^{-1} H^{-a}(\operatorname{Sol} \mathcal{M}) .
$$

Proof. By definition of the symbols involved ( ${ }^{\mathrm{p}} H$ denotes perverse cohomology, $\left.\operatorname{Sol}\left(\_\right) \stackrel{\text { def }}{=} \mathbf{R} \operatorname{Hom}_{\mathcal{D}_{X}}\left(\ldots, \mathcal{O}_{X}\right)[n]\right)$ we have

$$
\operatorname{Sol}\left(H_{[x]}^{a}(\mathcal{M})\right) \cong \operatorname{Sol} H^{a}\left(\mathbf{R} \Gamma_{[x]} \mathcal{M}\right) \cong{ }^{\mathrm{p}} H^{-a}\left(\operatorname{Sol}\left(\mathbf{R} \Gamma_{[x]} \mathcal{M}\right)\right) .
$$

\footnotetext{
${ }^{5}$ In order to recover our part (2) of Theorem 1.2 one proceeds as in GLS98 Remark 1]) and uses Poincaré duality for the link $L_{(Y, x)}$ of the singularity $(Y, x)$. The link is a real orientable compact manifold of dimension $2 d-1$. We have, locally analytically around $x$ that

$$
\begin{aligned}
H^{d+a-1}(Y-\{x\}, \mathbb{C}) & \cong H^{d+a-1}\left(L_{(Y, x)}, \mathbb{C}\right) \\
& \cong H^{d-a}\left(L_{(Y, x)}, \mathbb{C}\right) \quad \text { (Poincaré duality) } \\
& \cong H^{d-a}(Y-\{x\}, \mathbb{C}) .
\end{aligned}
$$

At the same time $H_{\{x\}}^{d+a}(Y, \mathbb{C}) \cong H^{d+a-1}(Y-\{x\}, \mathbb{C})$ and $H_{\{x\}}^{d-a+1}(Y, \mathbb{C}) \cong H^{d-a}(Y-$ $\{x\}, \mathbb{C})$ for $a \neq d$. When $a=d$ one has $\operatorname{dim}_{\mathbb{C}} H_{\{x\}}^{1}(Y, \mathbb{C})=\operatorname{dim}_{\mathbb{C}} H^{0}(Y-\{x\}, \mathbb{C})+1$ and the claim follows.
} 
One has that $\mathrm{Sol} \circ \mathbf{R} \Gamma_{[x]} \cong k_{!} k^{-1} \circ \operatorname{Sol}^{6}$ Thus ${ }^{\mathrm{P}} H^{-a}\left(\operatorname{Sol}\left(\mathbf{R} \Gamma_{[x]} \mathcal{M}\right)\right)$ is equal to

$$
{ }^{\mathrm{p}} H^{-a}\left(k_{!} k^{-1}(\operatorname{Sol}(\mathcal{M}))\right) \cong k_{!}^{\mathrm{p}} H^{-a}\left(k^{-1}(\operatorname{Sol}(\mathcal{M}))\right)
$$

where the isomorphism holds since $k_{\text {! }}$ is $t$-exact by [KW01, Lemma III.4.1]. After pullback along $k$ we are on the point $x$ on which perverse cohomology is the same as ordinary cohomology. Thus we may replace ${ }^{\mathrm{P}} H^{-a}$ by $H^{-a}$. Using that $k^{-1}$ is exact we get

$$
k_{!}^{\mathrm{p}} H^{-a}\left(k^{-1}(\operatorname{Sol}(\mathcal{M}))\right) \cong k_{!} H^{-a}\left(k^{-1}(\operatorname{Sol}(\mathcal{M}))\right) \cong k_{!} k^{-1} H^{-a}(\operatorname{Sol}(\mathcal{M}))
$$

as claimed.

One of our tools is the intersection homology $\mathcal{D}_{X}$-module $\mathcal{L}(Y, X)$ of Brylinski and Kashiwara BK81. It is the middle extension

$$
\mathcal{L}(Y, X) \cong \tilde{j}_{!_{*}} H_{[Y-\operatorname{Sing} Y]}^{n-d}\left(\mathcal{O}_{(X-\operatorname{Sing} Y)}\right)
$$

where $\tilde{j}$ denotes the open inclusion $(X-\operatorname{Sing} Y) \subseteq X$. Its characterizing property is that it is the smallest $\mathcal{D}_{X}$-submodule of $H_{[Y]}^{n-d}\left(\mathcal{O}_{X}\right)$ which agrees with $H_{[Y]}^{n-d}\left(\mathcal{O}_{X}\right)$ away from the singular locus of $Y$. Thus in particular if $Y$ is smooth then $\mathcal{L}(Y, X)=H_{[Y]}^{n-d}\left(\mathcal{O}_{X}\right)$.

Lemma 2.4. Let $X$ be a smooth $k$-variety of dimension n, let $i: Y \hookrightarrow X$ be a closed subvariety of dimension $d$ and assume that for $x \in Y$ one has $\left.\left.\mathcal{L}(Y, X)\right|_{(X-\{x\})} \cong H_{[Y]}^{n-d}\left(\mathcal{O}_{X}\right)\right|_{(X-\{x\})}$. Then

$$
H_{[x]}^{a}\left(H_{[Y]}^{n-d}\left(\mathcal{O}_{X}\right)\right) \cong H_{[x]}^{a}(\mathcal{L}(Y, X))
$$

for $a \geq 2$.

Proof. By assumption one has the short exact sequence

$$
0 \rightarrow \mathcal{L}(Y, X) \rightarrow H_{[Y]}^{n-d}\left(\mathcal{O}_{X}\right) \rightarrow \mathcal{C} \rightarrow 0
$$

whose cokernel $\mathcal{C}$ has support in the point $x$. Thus $H_{[x]}^{a}(\mathcal{C})=0$ for $a \geq 1$. By the long exact sequence of $H_{[x]}^{\bullet}\left(\_\right)$applied to this short exact sequence the claim of the lemma follows.

\footnotetext{
${ }^{6}$ This follows from the fact that for any closed embedding $k: Z \rightarrow X$ and complex of $\mathcal{D}_{X}$-modules $\mathcal{M}^{\bullet}$ one has the triangle

$$
\mathbf{R} \Gamma_{[Z]} \mathcal{M}^{\bullet} \rightarrow \mathcal{M}^{\bullet} \longrightarrow \mathbf{R} j_{*} j^{*} \mathcal{M}^{\bullet} \stackrel{+1}{\longrightarrow}
$$

where $j$ denotes the open inclusion $X-Z \subseteq X$. Let us denote $\operatorname{Sol}\left(\mathcal{M}^{\bullet}\right)$ by $\mathcal{L}^{\bullet}$ and apply Sol to this triangle. Using the compatibility of Sol with the six operations, in particular Sol $\circ j_{*} \cong j_{\text {! }} \circ$ Sol and Sol $\circ j^{-1} \cong j^{!} \circ$ Sol, we obtain the following triangle.

$$
\mathbf{R} j ! j^{!} \mathcal{L}^{\bullet} \longrightarrow \mathcal{L}^{\bullet} \longrightarrow \operatorname{Sol}\left(\mathbf{R} \Gamma_{[Z]} \mathcal{M}^{\bullet}\right) \stackrel{+1}{\longrightarrow}
$$

Comparing with the standard triangle $\mathbf{R} j ! j^{!} \mathcal{L}^{\bullet} \rightarrow \mathcal{L}^{\bullet} \longrightarrow \mathbf{R} k_{!} k^{-1} \mathcal{L}^{\bullet} \stackrel{+1}{\longrightarrow}$ KS90 Triangle 2.6.33] one obtains the claim.
} 
Lemma 2.5. Let $X$ be a smooth $k$-variety of dimension $n$ and let $Y \subseteq X$ be a closed subvariety of dimension d. Assume that for $x \in Y$ one has $\left.\left.\mathcal{L}(Y, X)\right|_{(X-\{x\})} \cong \mathbf{R} \Gamma_{[Y]}^{n-d}\left(\mathcal{O}_{X}\right)\right|_{(X-\{x\})}[n-d]$. Then

$$
\operatorname{Sol}(\mathcal{L}(Y, X)) \cong i_{!} j_{! *} \mathbb{C}_{(Y-\{x\})}[d]
$$

where $j$ is the inclusion of $(Y-\{x\}) \hookrightarrow Y$.

Proof. Let us fix the notation $(X-\operatorname{Sing} Y) \stackrel{j^{\prime \prime}}{\longrightarrow}(X-\{x\}) \stackrel{j^{\prime}}{\longrightarrow} X$. Then by definition of middle extension and our assumption one has

$$
\begin{aligned}
\mathcal{L}(Y, X) & \cong j_{!_{*}}^{\prime} j_{!_{*}}^{\prime \prime} H_{[Y-\operatorname{Sing} Y]}^{n-d}\left(\mathcal{O}_{(X-\operatorname{Sing} Y)}\right) \\
& \cong j_{!_{*}}^{\prime} \mathcal{L}(Y-\{x\}, X-\{x\}) \\
& \cong j_{!_{*}}^{\prime} \mathbf{R} \Gamma_{[Y-\{x\}]}\left(\mathcal{O}_{(X-\{x\})}\right)[n-d] .
\end{aligned}
$$

Denoting the inclusion $(Y-\{x\}) \hookrightarrow(X-\{x\})$ by $i^{\prime}$ we have (see footnote 5 )

$$
\begin{aligned}
\operatorname{Sol}\left(\mathbf{R} \Gamma_{[Y-\{x\}]}\left(\mathcal{O}_{(X-\{x\})}\right)[n-d]\right) & \cong i_{!}^{\prime} i^{\prime-1} \operatorname{Sol}\left(\mathcal{O}_{(X-\{x\})}\right)[d-n] \\
& \cong i_{!}^{\prime} i^{\prime-1} \mathbb{C}_{(X-\{x\})}[n][d-n]=i_{!}^{\prime} \mathbb{C}_{(Y-\{x\})}[d]
\end{aligned}
$$

where we also used that $\operatorname{Sol}\left(\mathcal{O}_{(X-\{x\})}\right) \cong \mathbb{C}_{(X-\{x\})}[n]$. Now finish the proof with the following chain of equalities

$$
\begin{aligned}
\operatorname{Sol}(\mathcal{L}(Y, X)) & \cong j_{!_{*}}^{\prime} \operatorname{Sol}\left(\mathbf{R} \Gamma_{[Y-\{x\}]}\left(\mathcal{O}_{(X-\{x\})}\right)[n-d]\right) \\
& \cong j_{!_{*}}^{\prime} i_{!}^{\prime} \mathbb{C}_{(Y-\{x\})}[d] \\
& \cong i_{!} j_{! *} \mathbb{C}_{(Y-\{x\})}[d]
\end{aligned}
$$

the last of which follows from the fact that for a closed immersion $i_{!} \cong i_{\text {!* }}$ and thus the $j$ and $i$ can be exchanged as we have done.

Remark 2.6. Granted, the assumption on $\mathcal{L}(Y, X)$ of the preceding two lemmata seems somewhat random. In characteristic zero (say over $\mathbb{C}$ ) they are equivalent via the Riemann-Hilbert correspondence to $(Y-\{x\})$ being an intersection cohomology manifold, see Mas. In positive characteristic our notion of close to $F$-rational of the final section relates it to previous work on singularities, such as tight closure theory and the notion of $F$-depth as in HS77.

Also note that if $Y$ has an isolated singularity at $x$ then the assumptions are (trivially) satisfied since in this case one has $\left.\mathcal{L}(Y, X)\right|_{(Y-\{x\})} \cong$ $H_{(Y-\{x\})}^{n-d}\left(\mathcal{O}_{(X-\{x\})}\right) \cong \mathbf{R} \Gamma_{[Y-\{x\}]}\left(\mathcal{O}_{(X-\{x\})}\right)[n-d]$.

Proof of Theorem 1.2. By assumption $(Y-\{x\})$ is an intersection homology manifold which in particular implies by Remark 2.1 that part (3) holds and part (1) and (2) are equivalent. Thus it is enough to show, say, part (2):

As we pointed out in the introduction the $\mathcal{D}_{X}$-module $H_{[x]}^{a} H_{[Y]}^{i}\left(\mathcal{O}_{X}\right)$ is isomorphic to a finite direct sum of $\lambda_{a, i}(A)$ many copies of $H_{[x]}^{n}\left(\mathcal{O}_{X}\right)$, 
the injective hull of the residue field at $x$. By Lemma 2.3 together with $\operatorname{Sol}\left(\mathcal{O}_{X}\right)=\mathbb{C}_{X}[n]$ one has

$$
\operatorname{Sol}\left(H_{[x]}^{n}\left(\mathcal{O}_{X}\right)\right) \cong k_{!} k^{-1} H^{-n}\left(\mathbb{C}_{X}[n]\right) \cong k_{!} \mathbb{C}_{x}
$$

where we recall that $k$ was just the inclusion of $x \hookrightarrow X$. Therefore $\lambda_{a, i}(A)$ is just the dimension of the fiber at $x$ of $\operatorname{Sol}\left(H_{[x]}^{a} H_{[Y]}^{i}\left(\mathcal{O}_{X}\right)\right)$. Thus, for $a \geq 2$ we can compute

$$
\begin{array}{rlr}
\lambda_{a, d}(A) & =e\left(H_{[x]}^{a}\left(H_{[Y]}^{n-d}\left(\mathcal{O}_{X}\right)\right)\right) \\
& =\operatorname{dim}_{\mathbb{C}}\left(\operatorname{Sol}\left(H_{[x]}^{a}\left(H_{[Y]}^{n-d}\left(\mathcal{O}_{X}\right)\right)\right)\right)_{x} & \\
& =\operatorname{dim}_{\mathbb{C}}\left(\operatorname{Sol}\left(H_{[x]}^{a}(\mathcal{L}(Y, X))\right)\right)_{x} \quad \text { (by Lemma 2.4) } \\
& =\operatorname{dim}_{\mathbb{C}}\left(k_{!} k^{-1} H^{-a}(\operatorname{Sol} \mathcal{L}(Y, X))\right)_{x} \quad \text { (by Lemma 2.3) } \\
& =\operatorname{dim}_{\mathbb{C}}\left(H^{-a}\left(i_{!} j_{! *} \mathbb{C}_{(Y-\{x\})}[d]\right)\right)_{x} \quad \text { (by Lemma 2.5) } \\
& =\operatorname{dim}_{\mathbb{C}}\left(H^{-a}\left(j_{! *} \mathbb{C}_{(Y-\{x\})}[d]\right)\right)_{x}
\end{array}
$$

where $i$ is the inclusion $Y \hookrightarrow X$ and $j$ denotes the inclusion $(Y-\{x\}) \subseteq Y$. Since $j$ is just the inclusion of the complement of a single point it follows that

$$
j_{! *} \mathbb{C}_{(Y-\{x\})}[d] \cong \tau_{\leq d-1} \mathbf{R} j_{*} \mathbb{C}_{(Y-\{x\})}[d]
$$

by Bor84, V.2.2 (2)]. By definition of Deligne's truncation $\tau_{\leq d-1}$ one has for $a \geq 1$

$$
\left(H^{d-a}\left(j_{! *} \mathbb{C}_{(Y-\{x\})}\right)\right)_{x} \cong\left(\mathbf{R}^{d-a} j_{*} \mathbb{C}_{(Y-\{x\})}\right)_{x} .
$$

Applying the following Lemma 2.7 we get for $2 \leq a \leq d-1$ that

$$
\lambda_{a, d}(A)=\operatorname{dim}_{\mathbb{C}}\left(\mathbf{R}^{d-a} j_{*} \mathbb{C}_{(Y-\{x\})}\right)_{x}=\operatorname{dim}_{\mathbb{C}} H_{\{x\}}^{d-a+1}(Y, \mathbb{C})
$$

and (for $a=d)$ that $\lambda_{d, d}(A)=H_{\{x\}}^{1}(Y, \mathbb{C})+1$.

Lemma 2.7. Let $Y$ be a variety and let $x \in Y$ be a closed point and $C$ be a constant sheaf on $Y$. Then

$$
\left(\mathbf{R}^{i} j_{*} j^{-1} C\right)_{x} \cong H_{\{x\}}^{i+1}(Y, C)
$$

for $i \geq 1$ and (for $i=0$ ) one has the short exact sequence

$$
0 \rightarrow C_{x} \rightarrow\left(\mathbf{R}^{0} j_{*} j^{-1} C\right)_{x} \rightarrow H_{\{x\}}^{1}(Y, C) \rightarrow 0 .
$$

Proof. For the open inclusion $j:(Y-\{x\}) \hookrightarrow Y$ consider the triangle

$$
\mathbf{R} \Gamma_{\{x\}} C \rightarrow C \rightarrow \mathbf{R} j_{*} j^{-1} C \stackrel{+1}{\longrightarrow}
$$

and take its fiber at the point $x$ to obtain the following triangle:

$$
\left(\mathbf{R} \Gamma_{\{x\}} C\right)_{x} \rightarrow C_{x} \rightarrow\left(\mathbf{R} j_{*} j^{-1} C\right)_{x} \stackrel{+1}{\longrightarrow}
$$


Since $H^{i}\left(C_{x}\right)=0$ for $i>0$ and $H_{\{x\}}^{0}(C)=0$ (since $C$ is a constant sheaf) the long exact sequence of cohomology for this triangle yields

$$
0 \rightarrow C_{x} \rightarrow\left(\mathbf{R}^{0} j_{*} j^{-1} C\right)_{x} \rightarrow\left(H_{\{x\}}^{1}(Y, C)\right)_{x} \rightarrow 0
$$

and for $i \geq 1$

$$
\left(R^{i} j_{*} j^{-1} C\right)_{x} \cong\left(H_{\{x\}}^{i+1}(C)\right)_{x} .
$$

But clearly since $H_{\{x\}}^{i+1}(C)$ is supported on $x$ we have $\left(H_{\{x\}}^{i+1}(C)\right)_{x} \cong H_{\{x\}}^{i+1}(Y, C)$ which finishes the proof.

\section{The CASE OF POSITIVE CHARACTERISTIC.}

We very briefly recall the setup of the correspondence of Emerton and Kisin and point out the relevant facts which will make clear that the in characteristic zero also works in positive characteristic.

3.1. Emerton-Kisin correspondence. Let $k$ be a field of positive characteristic $p$ and let $X$ be a smooth $k$-variety. In [EK04 Emerton and Kisin establish an anti-equivalence (on the level of derived categories) between constructible $\mathbb{Z} / p \mathbb{Z}$-sheaves on $X_{\text {ét }}$ on one hand and locally finitely generated unit $\mathcal{O}_{F, X}$-modules on the other. Their construction closely models the Riemann-Hilbert correspondence and underlies the same formalism - except that there is no duality available on either side of the correspondence. This leads to the defect that their anti-equivalence is compatible with only three of Grothendieck's six operations, namely with analogs of $f^{!}, f_{*}$ (denoted $f_{+}$ in [EK04] ) and $\stackrel{\mathbb{L}}{\otimes} \mathcal{O}_{F, X}$ on the $\mathcal{O}_{F, X}$-module side, which correspond to $f^{*}, f_{\text {! }}$ and $\stackrel{\mathbb{L}}{\otimes_{\mathbb{Z} / p \mathbb{Z}}}$ on the constructible étale $\mathbb{Z} / p \mathbb{Z}$ side.

We will recall the definition of $\mathcal{O}_{F, X}$-module and point out that the local cohomology modules are locally finitely generated as such, so that the formalism of Emerton-Kisin can be applied to our study of the numbers $\lambda_{a, i}$. For a nice introduction see [EK03]; or [EK04] for the most general theory.

Definition 3.1. A quasi-coherent $\mathcal{O}_{X}$-module $\mathcal{M}$ together with an $\mathcal{O}_{X^{-}}$ linear map

$$
\vartheta: F^{*} \mathcal{M} \rightarrow \mathcal{M}
$$

is called an $\mathcal{O}_{F, X}$-module. Here $F$ denotes the Frobenius morphism on $X$. If $\vartheta$ is an isomorphism, then $(\mathcal{M}, \vartheta)$ is called unit.

Locally, if $X=\operatorname{Spec} R$, an $\mathcal{O}_{X, F^{-}}$module is nothing but a module $M$ over the non-commutative ring

$$
R[F] \stackrel{\text { def }}{=} \frac{R\langle F\rangle}{\left(r^{p} F-F r \mid r \in R\right)} .
$$

Such $R[F]$-module is called finitely generated if it is just that: finitely generated as an $R[F]$-module. Thus we have the notion of locally finitely generated for $\mathcal{O}_{F, X}-$ modules. 
One of the key results of the theory (which was essentially proved by Lyubeznik in Lyu97) is that the category of locally finitely generated unit $R[F]$-modules is abelian, and that every such $\mathcal{M}$ has finite length in that category Lyu97, Theorem 3.2].

Example 3.2. Let $X=\operatorname{Spec} R$ be affine. Then, abusing the identification of $\mathcal{O}_{X}$-modules and $R$-modules one can write $F^{*} M=R^{(1)} \otimes_{R} M$ where $R^{(1)}$ is the $R-R$-bimodule with the usual left structure and the right structure via the Frobenius map. Thus one sees immediately that the natural map

$$
F^{*} R=R^{(1)} \otimes_{R} R \rightarrow R
$$

sending $a \otimes r$ to $a r^{p}$ is an isomorphism, showing that $R$ is a fg (finitely generated) unit $R[F]$-module.

Let $g \in R$ be an element and consider the localization $R_{g}$. The natural map

$$
F^{*} R_{g}=R^{(1)} \otimes_{R} R_{g} \rightarrow R_{g}
$$

has an inverse given by sending $r / t$ to $r t^{p-1} \otimes 1 / t$. $R_{g}$ is generated as an $R[F]$-module by $1 / g$. Thus again $R_{g}$ is a fg unit $R[F]$-module.

Since local cohomology modules $H_{I}^{i}(R)$ for $I$ an ideal of $R$ can be computed via Čech resolutions, whose entries are localizations of the type $R_{g}$, the aforementioned result that the category of fg unit $R[F]$-modules is abelian implies that local cohomology modules are fg unit.

These examples are a special instance of more general results showing that the cohomology with supports functors are defined in the category of locally finitely generated unit $\mathcal{O}_{F, X^{-}}$modules [EK04, Proposition 5.11.5]. If $\mathcal{M}^{\bullet}$ is a bounded complex of such modules then so is $\mathbf{R} \Gamma_{[Y]} \mathcal{M}^{\bullet}$ for $Y$ a closed subvariety of $X$ and one has the usual triangle

$$
\mathbf{R} \Gamma_{[Y]} \mathcal{M} \rightarrow \mathcal{M} \rightarrow j_{+} j^{!} \mathcal{M} \stackrel{+1}{\longrightarrow}
$$

where $j: X-Y \rightarrow X$ denotes the open inclusion.

The correspondence of Emerton and Kisin is between the derived category of bounded complexes of $\mathcal{O}_{F, X}$ modules whose cohomology is locally finitely generated unit,

$$
D_{l f g u}^{b}\left(\mathcal{O}_{F, X}\right) \stackrel{\text { Sol }}{\sim} D_{c}^{b}\left(X_{\text {ét }}, \mathbb{Z} / p \mathbb{Z}\right)
$$

and the derived category of bounded complexes of $\mathbb{Z} / p \mathbb{Z}$ sheaves with constructible cohomology on $X_{\text {ét }}$. Furthermore, they define functors $f^{!}, f_{+}$ and $\_\stackrel{\mathbb{L}}{\otimes}_{\mathcal{O}_{F, X}}$. They are not the same as (though closely related to) the functors of Grothendieck-Serre duality.

The canonical $t$-structure on the left induces via the anti-equivalence an exotic $t$-structure on $D_{c}^{b}\left(X_{\text {ét }}, \mathbb{Z} / p \mathbb{Z}\right)$, which in turn is just the $t$-structure for the middle perversity as described by Gabber Gab00. Thus one obtains a notion of perverse sheaves and thus of perverse cohomology. 
3.2. Intermediate extensions. There is a theory of intermediate extensions. If $j: U \rightarrow X$ is a locally closed immersion of smooth $k$-schemes and $\mathcal{M}$ a lfgu $\mathcal{O}_{F, U^{-}}$module, then its intermediate extension $j_{!_{+}} \mathcal{M}$ is defined as the smallest submodule $\mathcal{M}^{\prime} \subseteq H^{0}\left(j_{+} \mathcal{M}\right)$ such that $j^{!} \mathcal{M}^{\prime}=\mathcal{M}$. Furthermore,

$$
\operatorname{Sol}\left(j_{!_{+}}(\mathcal{M})\right) \cong j_{!_{*}} \operatorname{Sol}(\mathcal{M}) \stackrel{\text { def }}{=} \operatorname{Im}\left({ }^{\mathrm{P}} H^{0}\left(j_{!} \operatorname{Sol}(\mathcal{M})\right) \rightarrow{ }^{\mathrm{p}} H^{0}\left(j_{*} \operatorname{Sol}(\mathcal{M})\right)\right)
$$

so that the intermediate extension is compatible with the correspondence EK03, Section 4.3]. We will only apply this to $\mathcal{M}=H_{[Y-\operatorname{Sing} Y]}^{n-d}\left(\mathcal{O}_{(X-\operatorname{Sing} Y)}\right)$ for $Y$ a closed subset of $X$ and $j$ the open inclusion $(X-\operatorname{Sing} Y) \subseteq X$. In this case we get

$$
\mathcal{L}(Y, X) \stackrel{\text { def }}{=} j_{!_{+}} H_{[Y-\operatorname{Sing} Y]}^{n-d}\left(\mathcal{O}_{(X-\operatorname{Sing} Y)}\right) \subseteq H_{[Y]}^{n-d}\left(\mathcal{O}_{X}\right)
$$

as its unique simple submodule. This important special case was already obtained in Bli04. The key point in obtaining these results is the aforementioned fact that lfgu $\mathcal{O}_{F, X}$-modules have finite length.

The following proposition lists the properties of the theory which are needed to be able to transfer the proof of Theorem 1.2 to positive characteristic.

Proposition 3.3. (1) $\operatorname{Sol}\left(\mathcal{O}_{X}\right) \cong \mathbb{Z} / p \mathbb{Z}[n]$ where $n$ is the dimension of $X$.

(2) For a closed immersion of smooth $k$-schemes $k: Y \rightarrow X$ one has Sol $\circ \mathbf{R} \Gamma_{[Y]} \cong k_{!} k^{-1} \circ$ Sol.

(3) Let $k: Y \rightarrow X$ be a closed immersion of smooth schemes. Then $k_{\text {! }}$ is $t$-exact.

Proof. Part (1) is just Example 9.3.1 in EK04.

For part (2) note that $\mathbf{R} \Gamma_{[Y]} \mathcal{M}$ is defined via the triangle

$$
\mathbf{R} \Gamma_{[Y]} \mathcal{M} \rightarrow \mathcal{M} \rightarrow j_{+} j^{!} \mathcal{M} \stackrel{+1}{\longrightarrow}
$$

with $j: X-Y \rightarrow X$ denoting the open inclusion. Applying Sol and using the fact that Sol interchanges $j_{+}$with $j_{\text {! }}$ and $j$ ! with $j^{*}$ by [EK04, Proposition 9.3, Proposition 9.5] we compare with the triangle

$$
j ! j^{*} \operatorname{Sol}(\mathcal{M}) \rightarrow \operatorname{Sol}(\mathcal{M}) \rightarrow k_{!} k^{-1} \operatorname{Sol}(\mathcal{M}) \stackrel{+1}{\longrightarrow}
$$

in $D_{c}^{b}\left(X_{\text {ét }}, \mathbb{Z} / p \mathbb{Z}\right)$ to obtain the result.

Part (3) can be checked by hand (using Gabbers definition of the $t$ structure in Gab00), but also follows via the correspondence from the fact that $k_{+}$is exact by [EK04, Remark 3.4.1].

Proof of Theorem 1.1. The assumption of close to $F$-rational implies by Proposition 4.2 that $\left.\left.\mathcal{L}(Y, X)\right|_{(X-\{x\})} \cong \mathbf{R} \Gamma_{Y}\left(\mathcal{O}_{X}\right)\right|_{(X-\{x\})}[n-d]$. This means in particular that $\left.\left.\mathcal{L}(Y, X)\right|_{(X-\{x\})} \cong H_{Y}^{n-d}\left(\mathcal{O}_{X}\right)\right|_{(X-\{x\})}$ and $H_{Y}^{i}\left(\mathcal{O}_{X}\right)$ 
is supported in $x$ for $i \neq n-d$. Thus the vanishing condition (2.1) is satisfied and therefore (by Section 2.1) part (3) holds and part (1) and (2) are equivalent. Again we prove part (2) to finish the argument.

This is done by following the arguments in the preceding section step by step, working on the étale site and replacing $\mathbb{C}$ by $\mathbb{Z} / p \mathbb{Z}$ whenever appropriate. Here are some remarks on this task which finishes the proof.

(1) For Lemma 2.3 one uses that $\mathbf{R} \Gamma_{[x]}$ commutes with Sol in the way claimed. Furthermore we use that $k_{\text {! }}$ is $t$-exact. This is Proposition 3.3 part (2) and (3).

(2) As pointed out at the beginning of the proof the assumptions of Lemma 2.4 and Lemma 2.3 are satisfied. For Lemma 2.4 literally the same argument holds after the existence of the middle extension $\mathcal{L}(Y, X)$ is established as discussed above. The same remark applies to Lemma 2.5

(3) In the actual proof one should use that $\operatorname{Sol}\left(\mathcal{O}_{X}\right) \cong \mathbb{Z} / p \mathbb{Z}[n]$ and the discussed properties of middle extension, in particular its compatibility with Sol.

(4) Lemma 2.7 is even stated for general coefficients and the argument is valid for any $k$-variety with the étale topology. The only caveat is that we implicitly used excision in the last part; an étale version of which can be found in Mil80, Chapter 3, Proposition 1.27], for example. ${ }^{7}$

Remark 3.4. In positive characteristic a more direct proof of our main result is possible. One observes that the numbers $\lambda_{0, i}(A)$ for $i=1 \ldots d-1$ can be interpreted in terms of the action of the Frobenius on $H_{m}^{i}(A)$. Namely if $A=R / I$ the local cohomology module $H_{I}^{n-i}(R)$ is obtained from the local cohomology module $H_{m}^{i}(A)$ via a certain functor $\mathcal{H}_{R, A}$ (introduced and studied in Lyu97, Section 4, Example 4.8]). This functorial relationship

$$
H_{I}^{n-i}(R) \cong \mathcal{H}_{R, A}\left(H_{m}^{i}(A)\right)
$$

implies that $\lambda_{0, i}(A)=e\left(H_{I}^{n-i}(R)\right.$ ) (which is called the corank of $H_{I}^{n-i}(R)$ in Lyu97) is equal to the dimension of the Frobenius stable part of $H_{m}^{i}(A)$, by Lyu97, Proposition 4.10]

Finally, in [HS77, Theorem 2.5] the latter is determined to be equal to $\operatorname{dim}_{k} H_{\{x\}}^{i}\left(Y_{\text {ét }}, \mathbb{Z} / p \mathbb{Z}\right)$ as required.

\section{Close to $F$-Rational singularities.}

We finish this note with a brief discussion of a new class of singularities, called close to $F$-rational.

\footnotetext{
${ }^{7}$ As Brian Conrad pointed out to me, this use of excision is more subtle than we indicate here if $k$ is not separably closed. Thus to be correct we assume $k$ separably closed.
} 
Definition 4.1. Let $(A, m)$ be a local noetherian ring of dimension $d$. Let $H_{m}^{\bullet}(A)=\oplus H_{m}^{i}(A)$ be the local cohomolgy with support in $m$. Then $A$ is called close to $F$-rational if and only if

$$
H_{m}^{*}(A) / 0^{F}
$$

is simple as an $A[F]$-module, where $0^{F}$ denotes all the elements of $H_{m}^{*}(A)$ which are annihilated by a power of the Frobenius.

If $Y$ is a noetherian scheme then $Y$ is called close to $F$-rational if for all closed points $y \in Y$ the local ring $\mathcal{O}_{Y, y}$ is close to $F$-rational.

Recall that $F$-rationality of $A$ is equivalent to $H_{m}^{*}(A)$ being simple as an $A[F]$-module (at least if $A$ is excellent). This implies that an $F$-rational ring is close to $F$-rational. The obstruction to $F$-rationality is the tight closure of zero $0^{*}$ in $H_{m}^{*}(A)$ (see Hun96 for relevant notions from the theory of tight closure). Close to $F$-rational just means that this obstruction is, if not zero ( $F$-rational) it is at least $F$-nilpotent. Since one always has that $H_{m}^{d}(A) \neq 0^{*} 8$ it follows that $A$ is almost $F$-rational if and only if $H_{m}^{d}(A) / 0_{H_{m}^{d}(A)}^{F}$ is $A[F]$-simple and $H_{m}^{i}(A)$ is $F$-nilpotent for $i \neq d$.

The following characterization of close to $F$-rational singularities is the key point of our investigation.

Proposition 4.2. Let $Y$ be a subvariety of dimension $d$ embedded in $X$ which is a smooth $k$-variety of dimension $n$ (char $k=p>0$ ). Then $Y$ is close to F-rational if and only if

$$
\mathcal{L}(Y, X) \cong \mathbf{R} \Gamma_{[Y]}\left(\mathcal{O}_{X}\right)[n-d]
$$

where $\mathcal{L}(Y, X)$ denotes the unique simple unit $R[F]$ submodule of $H_{[Y]}^{c}\left(\mathcal{O}_{X}\right)$.

Proof. This is a slight extension of the main result in Bli04. Since by definition, close to $F$-rational is checked locally, we have to verify that for every point $y \in Y$ the local ring $(A, m)=\mathcal{O}_{Y, y}$ is close to $F$-rational if and only if $\mathcal{L}(A, R)=\mathbf{R} \Gamma_{I}(R)[n-d]$ where $R=\mathcal{O}_{X, x}$ such that $A=R / I$. For this reduction we used that $\mathcal{L}(Y, X)$ and $\mathbf{R} \Gamma_{[Y]} \mathcal{O}_{X}$ localize.

In this situation Bli04, Theorem 4.9] states that $\mathcal{L}(A, R)=H_{I}^{n-d}(R)$ if and only if $0^{*}=0^{F}$ holds in $H_{m}^{d}(A)$. This latter condition is equivalent to $H_{m}^{d}(A) / 0^{F}$ being $A[F]$-simple since $0^{*}$ is the maximal proper $R[F]$ submodule of $H_{m}^{d}(A)$. It remains to point out that $H_{I}^{n-i}(R)$ is zero if and only if $H_{m}^{i}(A)$ is $F$-nilpotent. This is because, in the notation of Lyu97, Example 4.8] we have

$$
H_{I}^{n-i}(R) \cong \mathcal{H}_{R, A}\left(H_{m}^{i}(A)\right) .
$$

By Lyu97, Section 4] one has $\mathcal{H}_{R, A}(\mathcal{M})=0$ if and only if the $A[F]$-module $\mathcal{M}$ is $F$-nilpotent. It follows that $H_{I}^{n-i}(R)=0$ if and only $H_{m}^{i}(A)$ is $F-$ nilpotent.

\footnotetext{
${ }^{8}$ For the versed in tight closure theory: existence of test elements is responsible for this, see for example Hun96.
} 
Remark 4.3. Close to $F$-rational singularities are related to the notion of $F$-depth as introduced by Hartshorne and Speiser in [HS77, page 60]. One can verify that if $A$ is close to $F$-rational then $F$-depth $A=\operatorname{dim} A$. This notion of $F$-depth is shown to be equal to the étale $\mathbb{Z} / p \mathbb{Z}$-depth.

Thus the notion of (close to) $F$-rational singularities yields a reasonable description of the class of varieties $Y \subseteq X$ for which $\mathcal{L}(Y, X) \cong$ $\mathbf{R} \Gamma_{[Y]}\left(\mathcal{O}_{X}\right)[n-d]$ and consequently Theorem 1.1] holds.

Remark 4.4. To see that close to $F$-rational does not imply $F$-rational one can consider the example of $A=k[x, y, z] /\left(x^{4}+y^{4}+z^{4}\right)$. This is not $F-$ rational but in Bli01, Example 5.28] the first author shows that it is close to $F$-rational precisely if the characteristic $p$ of $k$ is congruent to $3 \bmod 4$.

Remark 4.5. In a recent preprint Mas] Massey introduces and studies the notion of intersection homomolgy manifold in characteristic zero. As we pointed out before, this notion means precisely that

$$
\mathcal{L}(Y, X) \cong \mathbf{R} \Gamma_{[Y]}\left(\mathcal{O}_{X}\right)[n-d]
$$

whenever $Y \subseteq X$ is embedded into a smooth variety $X$. He gives several alternative characterisations, particularly for $Y$ a complete intersection.

Also in the complete intersection case Torrelli gives in [Tor] a characterization of $\mathcal{L}(Y, X) \cong \mathbf{R} \Gamma_{[Y]}\left(\mathcal{O}_{X}\right)[n-d]$ in terms of the Bernstein polynomials. In the case that $Y=(f=0)$ is a hypersurface his condition is easily phrased: The reduced Bernstein polynomial (that is divide the usual Bernstein polynomial by $(x+1))$ of $f$ has no integral root $\leq-1$.

\section{REFERENCES}

[BK81] J.-L. Brylinski and M. Kashiwara, Kazhdan-Lusztig conjecture and holonomic systems, Invent. Math. 64 (1981), no. 3, 387-410.

[Bli04] M. Blickle, The intersection homology D-module in finite characteristic, Math. Ann. 328 (2004), 425-450.

[Bli01] The intersection homology D-module in positive characteristic, Univ. of Michigan Dissertation, 2001.

[Bor84] A. Borel et al, Intersection cohomology, Progress in Mathematics, vol. 50, Birkhäuser Boston Inc., Boston, MA, 1984, Notes on the seminar held at the University of Bern, Bern, 1983, Swiss Seminars.

[EK03] M. Emerton and M. Kisin, An introduction to the Riemann-Hilbert correspondence for unit $\mathcal{F}$-crystals., Geometric aspects of Dwork theory. Vol. I, II, 677-700, Walter de Gruyter GmbH \& Co. KG, Berlin, 2004.

[EK04] _ Riemann-Hilbert correspondence for unit $\mathcal{F}$-crystals., Astrisque No. 293, (2004), vi+257 pp.

[Gab00] O. Gabber, Notes on some t-structures, handwritten notes, 2000.

[GLS98] R. Garca Lpez and C. Sabbah, Topological computation of local cohomology multiplicities, Collect. Math. 49 (1998), no. 2-3, 317-324, Dedicated to the memory of Fernando Serrano.

[HS77] R. Hartshorne and R. Speiser, Local cohomological dimension in characteristic p, Annals of Mathematics 105 (1977), 45-79.

[HS93] C. L. Huneke and Ro. Y. Sharp, Bass numbers of local cohomology modules, Trans. Amer. Math. Soc. 339 (1993), no. 2, 765-779. 
[Hun96] C. Huneke, Tight closure and its applications, Published for the Conference Board of the Mathematical Sciences, Washington, DC, 1996, With an appendix by Melvin Hochster.

[KS90] M. Kashiwara and P. Schapira, Sheaves on manifolds, Grundlehren der mathematischen Wissenschaften, vol. 292, Springer-Verlag, 1990.

[KW01] R. Kiehl and R. Weissauer, Weil conjectures, perverse sheaves and l'adic Fourier transform, Ergebnisse der Mathematik und ihrer Grenzgebiete. 3. Folge., vol. 42, Springer-Verlag, Berlin, 2001.

[Lyu93] G. Lyubeznik, Finiteness properties of local cohomology modules (an application of D-modules to commutative algebra), Invent. Math. 113 (1993), no. 1, 41-55.

[Lyu97] G. Lyubeznik, $\mathcal{F}$-modules: an application to local cohomology and D-modules in characteristic $p>0$, Journal für reine und angewandte Mathematik 491 (1997), 65-130.

[Mas] D. B. Massey, Intersection Cohomology, Monodromy, and the Milnor Fiber.

[Mil80] J. S. Milne, tale cohomology, Princeton University Press, Princeton, New Jersey, 1980.

[Ogu73] A. Ogus, Local cohomological dimension of algebraic varieties, Ann. of Math. (2) 98 (1973), 327-365.

[Tor] T. Torrelli, Intersection homology D-module and Bernstein polynomials associated with a complete intersection, preprint July 2004.

Universität Essen, FB6 Mathematik, 45117 Essen, Germany

E-mail address: manuel.blickle@uni-essen.de

$U R L$ : www.mabli.org

Universität Essen, FB6 Mathematik, 45117 Essen, Germany

E-mail address: raphael.bondu@uni-essen.de 